\title{
Cultura, identidad e imagen organizacional: desarrollo de una metodología para su estudio en la Universidad
}

Lucas J. Pujol-Cols y Mariana Foutel

\begin{abstract}
RESUMEN
La cultura, la identidad y la imagen organizacional constituyen tres partes interrelacionadas de un mismo sistema de construcción de significado que define a la organización en sus elementos más constitutivos. Los componentes de esta tríada conceptual constituyen variables críticas para la gestión universitaria al condicionar fuertemente los procesos involucrados en la adopción de decisiones estratégicas, tácticas y operativas. Con sustento en una revisión bibliográfica y en la experiencia adquirida por los autores a través de sus años de estudio del sistema universitario argentino, en este artículo se propone una metodología para el estudio de la cultura, la identidad y la imagen organizacional en una organización universitaria, proporcionando guías útiles tanto para investigadores interesados en el objeto de estudio como para actores involucrados en la gestión.
\end{abstract}

Palabras clave: cultura organizacional, identidad organizacional, imagen organizacional, educación superior, universidad, metodología, Argentina.

Lucas J. Pujol-Cols

lucaspujolcols@gmail.com

Argentino. Doctor en Administración (Universidad Nacional del Centro de la Provincia de Buenos Aires, Argentina), Master of Business Administration (MBA), Universidad Nacional de Mar del Plata, Argentina. Especialista en Docencia Universitaria. Investigador Asociado, Universidad Católica del Maule, Chile; Beca Doctoral de investigación del Consejo Nacional de Investigaciones Científicas y Técnicas (CONICET), Argentina. Temas de investigación: comportamiento organizacional y psicología laboral.

Mariana Foutel

marianafoutel@yahoo.com.ar Argentina. Magíster en Gerencia y Administración de Sistemas y Servicios de Salud, Universidad Favaloro, Argentina. Especialista en Administración de Negocios y en Docencia Universitaria. Profesora Titular y Directora del Departamento de Administración en la Facultad de Ciencias Económicas y Sociales de la Universidad Nacional de Mar del Plata, Argentina. Temas de investigación: management estratégico. 


\title{
Cultura, identidade e imagem organizacional: desenvolvimento de uma metodolo- gia para seu estudo na Universidade
}

\section{RESUMO}

A cultura, a identidade e a imagem organizacional constituem três partes inter-relacionadas de um mesmo sistema de construção de significado que define à organização em seus elementos mais essenciais. Os componentes desta tríada conceitual constituem variáveis críticas para a gestão universitária ao condicionar fortemente os processos envolvidos na adoção de decisões estratégicas, táticas e operativas. Com sustento numa revisão bibliográfica e na experiência adquirida pelos autores através de seus anos de estudo do sistema universitário argentino, neste artigo se propõe una metodologia para o estudo da cultura, a identidade e a imagem organizacional em uma organização universitária, proporcionando guias úteis tanto para investigadores interessados no objeto de estudo como para atores envolvidos na gestão.

Palavras chave: cultura organizacional, identidade organizacional, imagem organizacional, educação superior, universidade, metodologia.

\section{Culture, identity and organizational image: development of a methodology for its study at the University}

\begin{abstract}
Culture, identity and organizational image constitute three interrelated parts of the same meaning construction system that defines the organization in its most constitutive elements. The components of this conceptual triad are critical variables for university management by strongly conditioning the processes involved in the adoption of strategic, tactical and operational decisions. Based on a bibliographic review and on the experience acquired by the authors through their years of study of the Argentine university system, this article proposes a methodology for the study of culture, identity and organizational image in a university organization, providing useful guidelines for researchers interested in the object of study and for actors involved in higher education management.
\end{abstract}

Key words: organizational culture, organizational identity, organizational image, higher education, university, methodology.

Recepción: 17/08/16. Aprobación: 08/08/17. 


\section{Introducción}

Tal y como ha sido observado por Stensaker, Valimaa y Sarrico (2012), o más recientemente por Stensaker (2015), un volumen importante del interés académico generado en el campo de la educación superior pareciera encontrarse concentrado en el estudio de los aspectos intangibles de las organizaciones educativas, dentro de los cuales se incluyen la cultura, la identidad y la imagen organizacional. Estos fenómenos, que por definición constituyen tres partes interrelacionadas de un mismo sistema de construcción de significado (Hatch y Schultz, 1997), poseen una relevancia central para la gestión universitaria al constituir fuertes condicionantes tanto en la dinámica como en la efectividad de los procesos de toma de decisiones (Fumasoli y Stensaker, 2013).

Puesto que la cultura organizacional provee a los miembros de un marco de referencia socialmente compartido e inconsciente que los orienta en su forma de percibir, interpretar y reaccionar a los eventos que ocurren en la organización (Schein, 2010), también influye fuertemente en la conformación de la identidad y la imagen organizacional y, en definitiva, en la racionalidad individual, grupal y organizacional que subyace a la hora de pensar alternativas y sus restricciones (lo posible); proceder a su evaluación (lo mejor); definir qué aspectos deben ser priorizados (lo deseable) y cuáles deben ser evitados (lo prohibido); considerar qué actores deben ser partícipes del proceso, entre otros. Como resultado, una noción sistémica de la cultura organizacional y sus relaciones resulta fundamental para la gestión universitaria al favorecer: a) la adopción de decisiones que se encuentren sujetas al menor nivel posible de resistencia y antagonismo; b) la identificación de conflictos reales o potenciales desde una perspectiva de mayor complejidad que contemple sus relaciones con otros fenómenos que son parte de la vida organizacional; c) el reconocimiento de contradicciones productoras de tensiones en la organización; d) la compresión del significado simbólico de las decisiones, prácticas y comportamientos, y e) el entendimiento de las razones por las que varios grupos de integrantes poseen percepciones y representaciones diversas y, a menudo, opuestas de la realidad organizacional (Tierney, 2008; 1988).

Si bien el estudio de la cultura organizacional y sus relaciones con multiplicidad de fenómenos posee una larga tradición en la literatura del management (Giorgi, Lockwood y Glynn, 2016), dificultades apreciables se evidencian aun a la hora de lograr operacionalizaciones adecuadas que permitan su abordaje empírico (Martin, Frost, y O’Neill, 2006). Sackmann (1992) advierte que parte de tales obstáculos provienen de la notoria ambigüedad inherente al fenómeno, la profunda fragmentación conceptual presente en el campo y la concentración casi exclusiva de los investigadores en los aspectos más tangibles de la cultura organizacional, tales como los elementos fisicos, las prácticas, los procedimientos y las normas. Una situación similar ocurre con el estudio de los dos constructos restantes que son foco de este artículo, la identidad y la imagen organizacional, los cuales no se encuentran exentos de las ambigüedades comentadas previamente.

Con el principal propósito de facilitar e impulsar el desarrollo de futuras investigaciones en la temática, en este artículo se propone una metodología para el estudio de la cultura, la identidad y la imagen organizacional en la universidad, sustentada en una revisión de la literatura y en la experiencia adquirida por los autores como resultado de sus años de estudio del sistema universitario argentino. Se le propone al lector un recorrido compuesto por tres secciones. En la primera, se abordan las nociones de identidad, cultura e imagen organizacional desde el plano teórico y se presenta el denominado modelo de dinámicas culturales (Hatch y Schultz, 1997). En la segunda, se comparan estrategias para el abordaje de los tres fenómenos y se propone una metodología. En la tercera sección se discuten recomendaciones para practitioners o investigadores interesados. 


\section{Revisión de la literatura}

Tal y como sostienen Hatch y Schultz (2002), los conceptos de cultura, identidad e imagen organizacional constituyen tres partes interrelacionadas de un mismo sistema integrado de construcción de significado que define a la organización en sus elementos más constitutivos. La profunda interrelación subyacente entre estos tres fenómenos obliga a los investigadores a estudiarlos de manera integral, de modo de develar los complejos procesos involucrados entre ellos (Ravasi, 2016). En las siguientes líneas se precisará acerca de los tres fenómenos.

\section{La cultura organizacional}

Representa una de las fuerzas más complejas que opera al nivel de la organización. Es entendida por Schein (2010) como el patrón de presunciones básicas que un cierto grupo ha creado, descubierto o desarrollado al intentar dar respuesta a problemas tanto de adaptación externa como de integración interna, que ha demostrado funcionar lo suficientemente bien como para ser considerado válido $\mathrm{y}$, por tanto, ser enseñado a nuevos miembros como la manera correcta de percibir, pensar y sentir con relación a dichos problemas.

De acuerdo con el modelo de Schein (2010), la cultura de una organización existe en tres niveles, ordenados según su grado de superficialidad y visibilidad para el observador. El primero está dado por los artefactos, que son los elementos más visibles y tangibles de la cultura. Dentro de esta categoría es posible mencionar el espacio físico de trabajo, la tecnología, los códigos (formales o no formales) de vestimenta, los patrones observables de comportamiento, las historias (míticas o no), el material oficial de difusión, entre muchos otros. Aun cuando su existencia puede resultar aparente para el observador, Schein (2010) advierte que este nivel podría resultar engañoso por dos motivos: a) no todo elemento visible es cultural, sino que su creación podría responder a otras lógicas no necesariamente culturales (por ejemplo, políticas), b) la interpretación de cada uno de ellos suele resultar bastante más dificultosa que su mera observación.

El segundo nivel está dado por los valores que suelen gobernar el comportamiento humano dentro de la organización. Así, mientras que los artefactos responden al "qué ocurre", los valores pretenden dar respuesta al "por qué". Puesto que ellos no pueden ser observados en sí mismos, Schein (2010) sugiere que una manera de aproximarse al fenómeno consiste en la conducción de entrevistas a agentes clave de la organización, además del análisis de contenido y discursivo de artefactos.

Es frecuente que los sujetos se comporten de una cierta forma por razones que ellos mismos desconocen y que, probablemente, jamás se han cuestionado. Esta reproducción inconsciente de conductas es reflejo de lo que Schein (2010) ha denominado presunciones básicas subyacentes, constitutivas del núcleo más duro de la cultura organizacional y, en definitiva, su propia esencia. Así, y puesto que las presunciones básicas subyacentes suelen ser menos debatibles que los valores, el observador podría reconocerlas en aquellos casos en los que el entrevistado no sea capaz de explicar las razones por las que las cosas suceden de determinada manera por ser éstas demasiado obvias. El modelo propone que las presunciones básicas tienden a ir aglutinándose en patrones culturales, surgiendo, como resultado, un lenguaje y categorías comunes de percepción y pensamiento.

Hatch (1997) retoma y complejiza el clásico modelo de Schein (1984) incorporando dos modificaciones fundamentales en su denominado modelo de dinámicas culturales: a) identifica un cuarto elemento, los símbolos, dotados de un status superior dentro del sistema cultural, y b) se focaliza en las relaciones bidireccionales acaecidas entre los cuatro componentes de la cultura, atribuyéndole un rol menos protagonista al análisis de los elementos per sé. Desde el modelo de dinámicas culturales, los artefactos deben transformarse en símbolos si se pretende que 
sean aprehendidos como eventos, discursos u objetos culturalmente significativos.

La cultura organizacional puede ser abordada desde distintos niveles de análisis. Por un lado, puesto que representa un fenómeno colectivo, puede ser estudiada al nivel de grupos o de la organización en su conjunto. Pero además, y en la medida en la que constituye un marco para la construcción de sentido (contexto de significación) que orienta a los miembros en su percepción, interpretación y reacción a los eventos que acontecen en la organización, también resulta susceptible de ser analizada a nivel individual.

Con relación al primero de los niveles, Hofstede, Bond y Luk (1993) enfatizan en que la cultura representa una característica de la organización en su conjunto y no de los individuos que la componen. Así, señalan que mientras que los sujetos pueden ser removidos de la organización u otros convertirse en nuevos miembros, la cultura tiende a permanecer invariante. No obstante, reconocen que la manera más sencilla de abordarla es a través de las percepciones de los actores cuya experiencia transcurre en el marco de esa cultura.

Focalizándose exclusivamente en el nivel individual, Harris (1994) estudia la influencia de la cultura organizacional en los procesos y estructuras de sensemaking ${ }^{1}$ y en las respuestas subsecuentes. El autor entiende a la cultura organizacional como un sistema de esquemas que dirigen la manera en la que la información es adquirida y procesada, y el conocimiento es retenido y organizado. En palabras de Harris (1994), estos esquemas orientan a los individuos en su búsqueda de respuestas a cuatro preguntas: a) ¿quién/qué es éste/ esto?, b) ¿cuáles son sus implicaciones?, c) ¿qué significa? y d) ¿cómo debo responder?

Puesto que la cultura organizacional representa un fenómeno colectivo, ésta se ve reflejada en la emergencia de esquemas relativamente congruentes entre los miembros de una organización, cuyo contenido posee una saliencia similar, y que moldean y son moldeados por los procesos de sensemaking (Harris, 1994). A medida que los esquemas individuales se van volviendo más similares, la información que proveen a los sujetos tiende a volverse más enfocada, dirigida, clara, consistente y persuasiva. Como resultado, el conocimiento compartido (en este caso, cultural) se auto-perpetúa.

Schein (2010) señala que la cultura organizacional debe ser observada en lugar de medida, al estar compuesta por un conjunto de elementos subyacentes, casi invisibles, que definen formas compartidas, tácitas y dadas por sentado de percibir, sentir y reaccionar. A tales fines, recomienda los métodos clínicos o etnográficos, resaltando la necesidad de estar inmerso en el fenómeno y en la realidad organizacional bajo observación. En una misma línea se encuentra el planteo de Hatch y Schultz (1997: 357) cuando advierten que la cultura no debe ser vista por el investigador como una "variable a ser medida", sino como un contexto en el cual las interpretaciones de la identidad organizacional son socialmente construidas y las intenciones de influir en la imagen organizacional son formuladas. Las autoras abogan por una visión más compleja y dinámica de la cultura, la cual debe ser estudiada como parte de un sistema integrado en estrecha vinculación con la identidad y la imagen organizacional.

Aun cuando por los motivos descritos en el párrafo anterior se evidencia en la literatura el empleo predominante de técnicas cualitativas, también es posible identificar una gran cantidad de trabajos sustentados en metodologías cuantitativas (véase Schneider, Ehrhart y Macey, 2012, para una reciente revisión). En este sentido, si bien los diseños cualitativos de corte longitudinal e histórico-retrospectivos, basados principalmente en entrevistas en profundidad, talleres, revisión de fuentes documentales y

${ }^{1}$ El sensemaking refiere a una actividad por la cual los sujetos emplean sus estructuras cognitivas para percibir situaciones e interpretar tales percepciones. 
observación participante representan la tendencia predominante, los métodos cuantitativos pueden resultarle de utilidad al observador para dar mayor soporte o extender la información cualitativa relevada (Tierney, 2008).

\section{La identidad organizacional}

Para Albert y Whetten (1985), o más recientemente Whetten (2006), la identidad organizacional representa un entendimiento colectivo y comúnmente compartido acerca de los atributos más centrales, perdurables y distintivos de una organización. Refiere a lo que sus miembros perciben, sienten y piensan acerca de ella y sus aspectos más salientes (Hatch y Schultz, 1997). Para Corley, Harquail, Pratt, Glynn, Fiol y Hatch (2006), la identidad organizacional emerge de un proceso profundamente auto-reflexivo orientado a dar respuesta a dos interrogantes: “¿quiénes somos como organización?” y “ipor qué existimos?".

A diferencia de la cultura organizacional, la identidad representa un fenómeno eminentemente relacional (Ravasi y Schultz, 2006) que se construye como resultado de la influencia de dos procesos. Por un lado, y de la mano de la perspectiva del actor social, los managers contribuyen a la construcción de significado al intentar moldear las interpretaciones de los integrantes de la organización por medio de sus declaraciones formales (formal claims), proceso que recibe en la literatura el nombre de sensegiving. Por otro lado, y de la mano del constructivismo social, los agentes internos poseen libertad para negociar significados compartidos acerca de lo que la organización es y cuáles son sus elementos más distintivos, proceso definido oportunamente como sensemaking. Así, la identidad organizacional se construye como fruto del interjuego producido entre las afirmaciones identitarias (identity claims; es decir, lo que los miembros sostienen que la organización es) y los significados compartidos (identity understandings; es decir, lo que los miembros realmente creen que la organización es).
De manera contraria a lo usualmente sostenido en la literatura, Gioia, Patvardhan, Hamilton y Corley (2013) sostienen que la presunta estabilidad de la identidad organizacional resulta ilusoria al brindar la mayoría de la evidencia empírica soporte a la hipótesis contraria. Argumentan que la estabilidad aparente de la identidad organizacional se haya camuflada en una relativa invariancia de las etiquetas empleadas por los miembros de una organización para expresar lo que ellos creen que la organización es, pero que su significado asociado tiende a modificarse a través del tiempo. Los autores proponen que la identidad organizacional es un fenómeno mutable y dinámico que, como se verá más adelante, se encuentra sometido a revisiones y renegociaciones.

De igual modo en que se planteó para la cultura organizacional, para el estudio de la identidad organizacional suelen privilegiarse los diseños cualitativos de corte longitudinal, como lo son los estudios de caso, los estudios de casos múltiples o los estudios etnográficos, basados principalmente en la conducción de entrevistas en profundidad, en la revisión de fuentes documentales y en la observación participante (c.f. Ravasi y Schultz, 2006).

\section{La imagen organizacional}

El tercer elemento de esta tríada conceptual, la imagen organizacional, ha sido estudiado tanto por la literatura organizacional como la del marketing desde ópticas diferentes (Ravasi, 2016). Tal y como sostienen Hatch y Schultz (2002), la literatura del marketing se ha concentrado casi exclusivamente en una perspectiva externa de la imagen organizacional, definiéndola como las creencias y sentimientos acerca de una organización que se encuentran en la mente de sus variados públicos, tales como los clientes, la comunidad, los proveedores, los distribuidores, el Estado, las entidades reguladoras y de acreditación, los inversores, etcétera. Como resultado, esta visión externa de la imagen organizacional se focaliza en la impresión que la compañía genera en el entorno, 
concepto similar al de marca corporativa (corporate brand) o imagen corporativa (corporate image).

Menos documentado aún se encuentra el estudio de la perspectiva interna de la imagen organizacional, la cual puede ser definida como la manera en la que los miembros de una organización creen que ésta es vista por otros agentes externos (Dutton y Dukerich, 1991). Esta noción interna de lo que los otros piensan se construye como resultado de la interacción que diariamente existe entre agentes internos y externos así como de los intentos deliberados de los managers de influir en la opinión pública (Gioia, Hamilton y Patvardhan, 2014). En este sentido, Gioia et al. (2000) señalan que mientras que la identidad organizacional responde al interrogante de “quiénes somos como organización?", la imagen organizacional responde a "¿quiénes creen ellos que somos? Es desde esta última perspectiva que la imagen organizacional será analizada en este artículo.

\section{Relaciones identidad-cultura-imagen}

Ravasi (2016) advierte que la cultura, la identidad y la imagen organizacional requieren ser estudiadas en conjunto, de modo de lograr una comprensión más cabal de las relaciones que acaecen entre ellas. Modelos más estáticos resultan insuficientes para entender la compleja dinámica que subyace entre estos tres fenómenos. Para Hatch y Schultz (2002), son cuatro los procesos que los vinculan: a) expressing, b) mirroring, c) impressing, y d) reflecting.

Como se mencionó previamente, la identidad organizacional emerge de la interacción social ocurrida entre los miembros de una organización (sensemaking) y, a la vez, se ve influida por las declaraciones oficiales de la Dirección (sensegiving). Al mismo tiempo, la identidad se construye en el marco de una cultura dentro de la cual se encuentra embebida (Hatch y Schultz, 1997). Así, el repositorio cultural que cada agente posee le permite orientarse en la interpretación de los distintos eventos que acontecen y dotarlos de significado (Harris, 1994). En este sentido, la cultura organizacional le brinda a los miembros pistas que presionan hacia la construcción de un entendimiento compartido acerca los atributos más centrales, perdurables y distintivos de la organización. Lo anterior responde al primero de los procesos mencionados en el párrafo anterior, el expressing. Pero, al margen de que la identidad organizacional refleja entendimientos de origen cultural, ésta también se construye (o modifica) reflejando (mirroring) la percepción que los integrantes poseen de la mirada de los agentes externos, nutriéndose así de la influencia de la imagen organizacional. A su vez, la identidad organizacional tiende a expresarse fuera de las fronteras de la organización mediante la interacción entre agentes internos y externos y, en consecuencia, a dejar una impresión (impressing) en los otros. Finalmente, y aun cuando la cultura organizacional posee un núcleo duro difícilmente modificable (es decir, la esencia cultural) y propiedades auto-perpetuantes que la dotan de cierta estabilidad relativa, nuevos patrones de entendimiento colectivo tienden a ir cristalizándose, proceso denominado reflecting.

En cuanto a la dinámica de las relaciones entre la identidad y la cultura organizacional, Ravasi y Schultz (2006) advierten que, aun cuando la primera suele estar sujeta a revisiones, las mismas suelen realizarse en el marco de la cultura organizacional dentro de la cual ésta se halla embebida. En efecto, los autores sostienen que cuando un cambio es percibido por los miembros de una organización como amenazador a la identidad (identity threat), la historia construida a través de la experiencia compartida, los símbolos organizacionales y las prácticas consolidadas (parte de la cultura organizacional) les brindan orientación en su búsqueda de entendimiento sobre quiénes son como organización y cuál es su propósito. Así, tal y como ha sido sugerido previamente por Humphreys y Brown (2002), resulta altamente probable que los miembros tiendan a rechazar nuevas conceptualizaciones que resulten incoherentes con la cultura organizacional. 


\section{Desarrollo}

\section{Metodología propuesta}

De manera coincidente con el enfoque predominante en la literatura, la construcción de un diseño metodológico que combine el empleo de distintas estrategias cualitativas que le permitan al observador ${ }^{2}$ adentrarse lo más posible en la realidad organizacional (Tierney, 2008) se considera la perspectiva más apropiada para el estudio de la identidad, la cultura y la imagen organizacional de una universidad. No obstante, la utilización de técnicas cuantitativas puede resultar útil en aquellos casos en los que se desee relevar información sintética o identificar tendencias en el comportamiento de ciertas variables. La adopción de un enfoque clínico y etnográfico (Schein, 2010) le permitirá al observador comprender e interpretar el significado de los artefactos e ir rastreando poco a poco los elementos más tácitos y subyacentes del sistema cultural y de significado de la organización.

A la complejidad propia de los tres fenómenos que son foco de este artículo, se le adiciona la correspondiente a la universidad como fenotipo organizacional. ${ }^{3}$ Aun cuando se admiten variaciones según el país y su modelo institucionalizado de gestión, la universidad suele componerse por un gran número de participantes y grupos de participantes, cuyas racionalidades se sustentan en valores, objetivos e intereses no necesariamente compartidos. En líneas generales, es posible distinguir tres grandes grupos de participantes en la universidad: los docentes (incluidos dentro de esta categoría, los investigadores), los estudiantes y el personal de apoyo. ${ }^{4}$ Podría admitirse la identificación por separado de un cuarto grupo de individuos que se encarga de la Dirección de la universidad, cuya composición varía sustancialmente según las características de sistema universitario de cada país.

Para el estudio de estos tres fenómenos y de sus relaciones, se desarrolla una metodología de abordaje sustentada en el supuesto de que el observador desconoce completamente la realidad organizacional a ser analizada (véase figura 1). El empleo de ciertas herramientas aquí sugeridas podría ser omitido cuando existiera un mayor nivel de contacto o inmersión del observador en la organización. Sin embargo, se asume el riesgo de no ser capaz de ver lo que en realidad ocurre y que los propios prejuicios sesguen las interpretaciones.

La metodología que aquí se presenta se compone por tres grandes etapas:

1. Estudio cualitativo exploratorio, mediante el empleo de talleres, observación participante, revisión de fuentes documentales y focus groups.

\section{Estudio cuantitativo.}

3. Estudio cualitativo explication, a través de la conducción de entrevistas grupales e individuales.

\section{La observación participante}

La observación le permite al agente interesado tomar un primer contacto con la realidad organizacional bajo estudio y, a la vez, apreciar la manera en la que interaccionan los miembros en su ambiente natural de trabajo. Resulta particularmente útil para reconocer los elementos más superficiales y tangibles de la cultura organizacional. Sin embargo, enfatizan Fraire y Scribano (2008: 56), la observación requiere de la planificación de su aplicación para evitar que se convierta "en una mera mirada global de la realidad".

\footnotetext{
${ }^{2}$ Se emplea el término observador para referirse a la persona abocada al estudio de los fenómenos involucrados en este artículo, entendiendo que el mismo puede ser tanto un investigador como un practitioner (gestor de una organización educativa).

${ }^{3}$ Se entiende por fenotipo organizacional al conjunto de organizaciones que comparten rasgos similares en términos de su estructura, tecnología y ambiente.

${ }^{4}$ El término personal de apoyo se emplea para referirse al grupo de participantes abocados al desempeño de funciones que brindan soporte al flujo de actividades sustantivas de la universidad, como lo son la docencia, la investigación y la transferencia tecnológica. Dentro de estos es posible encontrar: personal de maestranza, personal administrativo, personal de soporte técnico, entre otros.
} 


\section{Figura 1. Estrategias metodológicas para el estudio de la cultura, la identidad y la imagen organizacional}

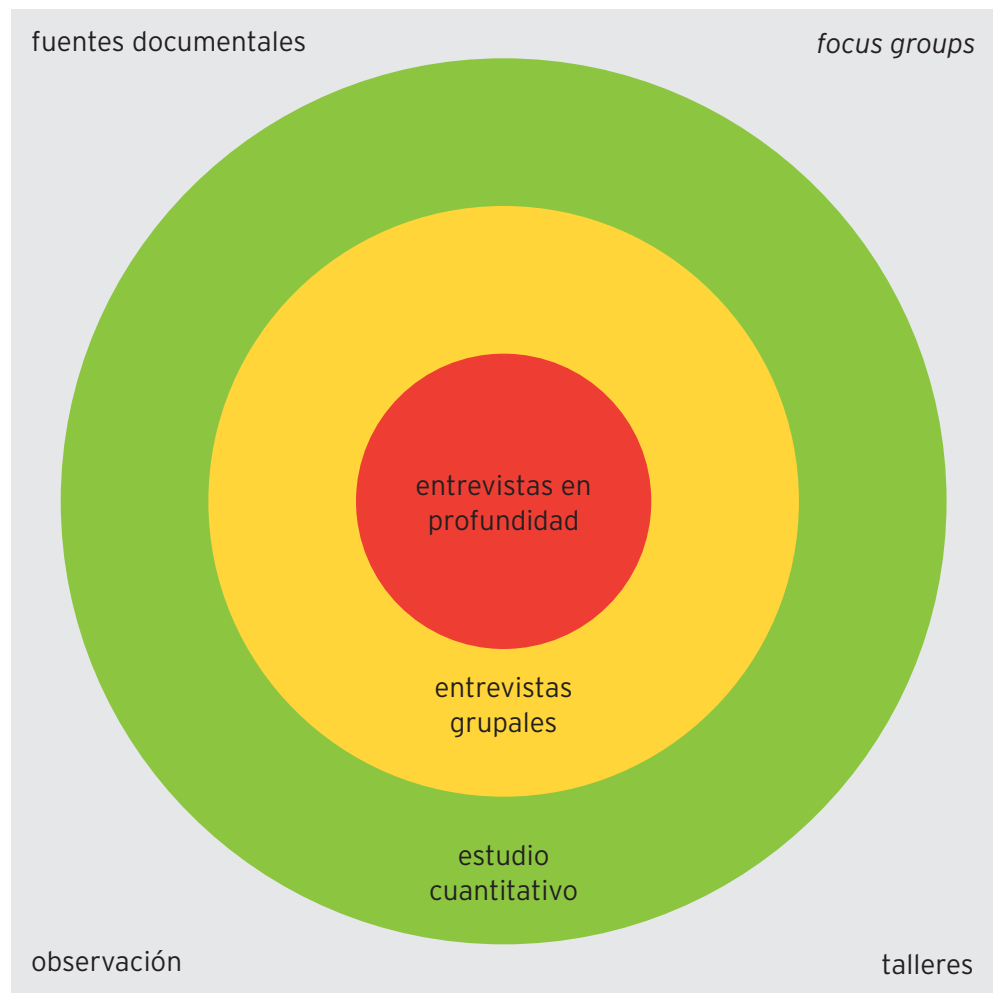

Algunos de los artefactos más visiblemente observables de la universidad, como las características del espacio (por ejemplo, la dimensión, el color, la distribución de los objetos y sectores, la señalización), las reglas (por ejemplo, las obligaciones establecidas en el estatuto para cada uno de los actores de la comunidad académica), los procedimientos (por ejemplo, la solicitud de una licencia), los mecanismos formales (por ejemplo, los mecanismos formales de participación en la toma de decisiones), los mitos (por ejemplo, los relatos sobre debates suscitados entre actores clave en la creación de una carrera o en la renovación de un plan de estudio) o los rituales (por ejemplo, un acto de inicio de ciclo lectivo, una ceremonia de graduación y entrega de distinciones a graduados sobresalientes, un acto de conmemoración a docentes con cierta cantidad de años de antigüedad, una ceremonia de reconocimiento a profesores eméritos), pueden ser identificados mediante la observación participante complementada con la revisión de fuentes documentales. Sin embargo, y como se verá en acápites posteriores, existen ocasiones en las que el observador deberá triangular las fuentes de información anteriores con otras herramientas. Lo anterior resulta particularmente cierto para el caso de otros artefactos de visibilidad menos aparente como lo son los comportamientos grupales y las prácticas o formas usuales de hacer las cosas dentro de la universidad.

\section{La revisión de fuentes documentales}

Si bien, como sugiere Schein (2010), los elementos más ocultos del sistema cultural de una organización tienden a ser expresados en un conjunto de artefactos y símbolos, ello puede ocurrir como resultado de 
un proceso inconsciente o bien como producto de intentos deliberados de la Dirección de perpetuar valores organizacionalmente deseables. Así, suelen existir diversas fuentes documentales a las que el observador puede tener acceso, entre ellas: a) el estatuto de constitución de la universidad, donde generalmente se encuentra la declaración de su misión y sus principios filosóficos; b) revistas institucionales o material de difusión interna y externa; c) mensajes institucionales enviados por correo electrónico o intranet a los miembros (por ejemplo, el Boletín de Novedades); d) mensajes institucionales emitidos a la comunidad (por ejemplo, artículos publicados en periódicos, participación de miembros de la Dirección en programas televisivos, videos institucionales); e) resoluciones de órganos de Gobierno.

Implicancias para la cultura organizacional. La organización surge en un ambiente específico y, como tal, la conformación y evolución de su cultura se ve influida por la cultura local y nacional, la tecnología, el contexto institucional, entre otros factores (Deal y Kennedy, 1982). La revisión de fuentes documentales puede orientar al observador en su comprensión del ambiente en el cual ha surgido, se ha desenvuelto y opera actualmente la organización bajo estudio. A su vez, le permite: la identificación preliminar de informantes clave y el reconocimiento de valores organizacionalmente deseables.

Implicancias para la identidad organizacional. El examen del estatuto de constitución le permite al observador reconocer la misión y principios filosóficos que la universidad proclama que guían su accionar. En términos generales, la misión constituye una declaración de la razón de ser de una organización. Su construcción se remonta a la época fundacional y suele gozar de relativa estabilidad a través del tiempo. La misión, así como el conjunto de principios filosóficos asociados, constituye el primer mensaje declarativo con contenido identitario (identity claim) de la Dirección a sus integrantes y uno de sus primeros intentos de influir en la construcción de sentido compartido en torno al interrogante de "¿por qué existimos?". De ella se desprende el conjunto de valores centrales que la organización desea preservar y transmitir de manera tal que se transformen en verdaderas guías para el comportamiento dentro de la misma. En definitiva, la misión y su transmisión asumen un rol fundamental en los procesos de sensegiving y sensemaking discutidos en secciones previas de este artículo.

Una de las primeras cuestiones que un investigador abocado al estudio de la cultura, la identidad y la imagen organizacional debe hacer es identificar: a) la declaración de la misión; b) los mecanismos organizacionalmente creados para asegurar su reproducción; c) la percepción que los integrantes poseen de la razón de ser de la organización, independientemente de las intenciones de la Dirección de influir en su opinión; d) el grado de congruencia existente entre el entendimiento compartido de la misión y el mensaje formal. Para ello, el observador podría considerar útil la triangulación de tres fuentes básicas de información: a) la revisión de fuentes documentales (por ejemplo, el estatuto, el material de comunicación interna y difusión externa), b) la conducción de entrevistas a miembros de la Dirección y c) la conducción de entrevistas a otros integrantes de la organización. Las dos últimas estrategias se profundizarán en apartados siguientes.

La información obtenida a través del análisis de la misión debe ser complementada con el examen de otras fuentes documentales, tales como las comunicaciones institucionales de difusión interna. Las anteriores también pueden ser concebidas como intentos deliberados de la Dirección de influir en los procesos de construcción de sentido.

Implicancias para la imagen organizacional. Tal y como sostienen Hatch y Schultz (1997), la Dirección suele realizar intentos frecuentes de influir en la opinión que los agentes externos poseen de la organización. Muchos de aquellos intentos suelen constar en mensajes transmitidos a través de medios masivos de comunicación y cuentas oficiales de la universidad 
en las redes sociales. El análisis de fuentes como las anteriores resulta necesario con el fin de diagnosticar la imagen que la Dirección desea brindar a la comunidad (imagen deseada).

\section{Los talleres}

Son una herramienta de gran utilidad para reunir evidencia exploratoria que oriente al observador en el empleo posterior de otras estrategias. Constituyen una adaptación de los clásicos grupos de discusión, semejantes a las "reuniones de vecinos" mencionadas por Fraire y Scribano (2008) y, al igual que los focus groups, ofrecen la posibilidad de reproducir (al menos artificialmente) la interacción entre sujetos, de modo de observar y registrar las manifestaciones y relaciones que se establecen dentro y entre grupos sociales. A diferencia de los focus groups, suelen caracterizarse por un menor nivel de estructuración y formalidad (Fraire y Scribano, 2008).

Aplicados al ámbito organizacional, suelen realizarse con la participación de entre 20 y 25 miembros provenientes de diferentes unidades que, agrupados en equipos de cuatro y cinco individuos, reflexionan en torno a un conjunto de tópicos disparadores pensados ex ante bajo la moderación de un experto (usualmente el investigador). Los participantes son seleccionados para captar la opinión social sobre la cultura, la identidad y la imagen organizacional. Resulta útil que el moderador circule entre los grupos de trabajo de modo tal que pueda observar y tomar notas sobre la manera en la que interactúan sus integrantes. Al respecto, Fraire y Scribano (2008) advierten que todo grupo individualmente considerado ha de combinar mínimos de homogeneidad y heterogeneidad. La primera para mantener la simetría de la relación de los componentes del grupo; la segunda para asegurar la diferencia necesaria en todo proceso de habla.

Finalizada la dinámica, debería preverse un espacio de intercambio y síntesis en el que representantes de cada equipo puedan compartir con el resto sus reflexiones. Lo anterior posibilita la identificación, aunque preliminar, de potenciales diferencias de percepción entre los distintos subgrupos sociales.

\section{Estudio cuantitativo}

La información relevada a través de la revisión de fuentes documentales, la observación participante y la conducción de talleres le brinda al observador orientación en la construcción del cuestionario. Esta etapa resulta sumamente útil pues contribuye a identificar tendencias en el comportamiento de ciertas variables, explorar diferencias entre subgrupos sociales, obtener indicadores de síntesis y lograr generalizaciones atribuibles al conjunto poblacional. Debido a la complejidad propia de los constructos involucrados, resulta deseable un diseño semi-estructurado que permita la incorporación tanto de preguntas cerradas (para identificar tendencias o diferencias entre grupos) como de preguntas abiertas (para posibilitar la emergencia de elementos no pensados y obtener evidencia que pueda ser triangulada con la obtenida a través de otras técnicas). El lector podrá encontrar un modelo de cuestionario en la sección Anexo de este artículo, el cual podría admitir adaptaciones dependiendo de las necesidades específicas de relevamiento.

Implicancias para la cultura organizacional. La experiencia compartida por los miembros en el espacio de trabajo a través de los años da lugar a la emergencia de historias que, de adquirir el status simbólico apropiado, pueden convertirse en verdaderos mitos organizacionales. Independientemente de su naturaleza verídica o no, estos relatos asumen un rol clave en la socialización de nuevos miembros. En la identificación de mitos podría ser adecuado consultar tanto a miembros de mayor como de menor antigüedad sobre si recuerdan algún hecho que haya marcado la historia de la universidad (item P1 del cuestionario disponible en el Anexo). A su vez, podría ser interesante preguntarles de qué manera han tomado conocimiento de aquellas historias, es decir, si ellos 
mismos las han presenciado (item P2) o si, por el contrario, las han conocido por medio de fuentes documentales, por su interacción con compañeros de trabajo (socialización), a través de comunicaciones institucionales, entre otros (item P3).

En términos de Schein (2010), detrás de los artefactos (y los símbolos) subyacen un conjunto de valores y presunciones que guían el comportamiento de los individuos dentro de la organización. Primeramente, y para motivar una emergencia espontánea de los mismos, se le podría solicitar al encuestado que enuncie hasta cinco valores que considera que tienen influencia en la toma de decisiones de la organización (item P4). Aquí se analizará fundamentalmente la saliencia (es decir, la frecuencia de evocación, cantidad de veces que el atributo es mencionado por los encuestados) y la centralidad (lugar que ocupa el atributo en la mente del sujeto: primera mención, segunda mención, tercera mención, etcétera). Acto seguido, se le podría entregar al participante un listado de atributos y solicitarle que indique, desde su percepción, el grado de presencia de cada uno de ellos en la universidad bajo análisis (item P5). Se advierte que es probable que, si existieran intentos frecuentes de la Dirección de reforzar valores organizacionalmente deseables, la respuesta de los encuestados se encuentre más alineada con dichas influencias.

Como se ha establecido en acápites precedentes, la cultura organizacional ejerce un rol fundamental, al moldear la percepción de los miembros de modo tal que exhiba un cierto nivel de congruencia. Sin embargo, lo anterior no significa suponer la existencia de un grado de convergencia absoluto. En efecto, la cultura organizacional puede ser más o menos compartida, posibilitando la existencia de culturas más fuertes o débiles (con una menor o mayor presencia de subculturas; Schein, 2010) y de patrones culturales dominantes o contrapuestos (contra-culturas). Como resultado, los valores y principios imperantes en el sistema cultural podrían no ser compartidos en la misma medida por todos los miembros o todos los subgrupos sociales. De manera coincidente con el planteo de Ribes Pons (1997), resulta interesante conocer los grados de coherencia y consistencia que presenta una determinada cultura organizacional pues permite identificar contradicciones internas y saber hasta qué punto están asumidas y compartidas las variables culturales de una organización por sus miembros.

La existencia de divergencias resulta un factor clave en la propensión al cambio de un determinado sistema cultural y suele materializarse en la intención de determinados actores de efectuar acciones que, ulteriormente, tengan fuerza instituyente. Como resultado, podría ser útil solicitarle a los entrevistados que listen un conjunto de principios que ellos entienden que guían (dominan) el comportamiento de la universidad pero que ellos no comparten (item P6) y que obstaculizan el cumplimiento de la misión (item P7). En este último caso debería pedírseles que brinden ejemplificación de algún caso concreto que dé soporte a su respuesta (ítem P8).

Como se ha sugerido, los componentes culturales de una organización se encuentran en continuo movimiento y son el resultado de una síntesis de antagonismos. Sin embargo, y como también se ha enfatizado, resulta esperable que los valores tiendan a poseer una cierta estabilidad dentro del sistema cultural. Así, podría ser apropiado solicitar a los participantes que listen al menos tres principios que, a su parecer, hayan permanecido más invariantes a través del tiempo (item P10) y tres que hayan afrontado mayores cambios (item P1 1). Luego de haber reunido evidencia espontánea, podría entregárseles un listado de atributos sugeridos para los que deban indicar un grado percibido de permanencia en el tiempo (item P12).

En los mitos suelen aparecer personajes destacados denominados héroes (Deal y Kennedy, 1982). Los héroes forman parte de la cultura organizacional en la medida en que son representativos de valores 
grupal u organizacionalmente deseables y, como tales, poseen una gran capacidad de influencia sobre el resto de los miembros. En nuestro caso de estudio, el observador podría pedirle a los participantes piensen en alguna persona a quien ellos consideren haya sido (o sea) representativa de alguno de los valores previamente enunciados (item P9).

Finalmente, el observador podría aprovechar la instancia para reunir evidencia exploratoria referida a subculturas y los criterios que determinan su conformación. A tales fines, se puede solicitar a los encuestados que indiquen las razones que, desde su percepción, motivan la aparición de subgrupos sociales dentro de la universidad (item P15). Otro enfoque apropiado podría ser efectuar cruces de información empleando los datos de clasificación de los participantes.

Implicancias para la identidad organizacional. Parte de la información discutida en el acápite anterior también brinda al observador evidencia útil para el estudio de la identidad organizacional. Con el fin de evitar reiteraciones, se destacan a continuación los puntos principales que le brindarán orientación en el análisis de la identidad de la universidad.

1. Atributos característicos de la universidad estudiada (evidencia espontánea y sugerida; items P4 y P5)

2. Principios característicos de la universidad que el encuestado no comparte (ítem P6),

3. Principios característicos que contribuyen al cumplimiento de la misión (ítems P7 y P8),

4. Análisis del grado de invariancia de los atributos más característicos de la universidad estudiada a través del tiempo (ítems P10, P11 y P12).

Implicancias para la imagen organizacional. En primer lugar, el observador debería preguntarle a los participantes sobre quiénes consideran ellos son agentes externos clave con influencia en el desarrollo de las actividades de la universidad (item P13). Luego, que indiquen su percepción de la imagen que aquellos tienen de la universidad. Con relación a este último aspecto, se recomienda descomponer esta evaluación en un conjunto de indicadores relevantes (item P14).

\section{Los focus groups}

Técnica usualmente empleada en instancias exploratorias de investigaciones de diseño cualitativo o mixto, dada su elevada potencialidad para permitir la emergencia espontánea de categorías como fruto del análisis de la interacción acaecida entre los participantes. Consiste en una discusión promovida y conducida por un moderador, quien actúa como facilitador de los intercambios que se suscitan alrededor de una o varias temáticas propuestas por éste (Fraire y Scribano, 2008). Se distingue de otro tipo de entrevistas grupales, ya que en este caso se busca promover la interacción entre los participantes de modo que construyan colaborativamente sus argumentos sobre la base de los aportes individuales. Consiste en un grupo de entre siete y nueve personas, seleccionadas de acuerdo a una serie de criterios relevantes, que dan respuesta a un conjunto de tópicos o preguntas sugeridas por un moderador.

Para evitar condicionamientos en las respuestas, el rol del moderador debería limitarse a: a) sugerir temáticas disparadoras contenidas en un guión, b) brindar clarificación ante requerimientos puntuales de los participantes, c) solicitar a los participantes que amplíen su discurso o profundicen en determinado tema, d) reencauzar la dinámica en caso de que la misma se escape de los objetivos del estudio. En todos los casos deberá procurarse que los participantes no se conozcan entre sí ni con los moderadores.

Para el caso concreto de la universidad, se sugiere la conducción de, al menos, dos dinámicas (una testigo y otra de control) por cada segmento o subsegmento que se desee analizar (por ejemplo, docentes, estudiantes, personal administrativo). La duración de cada una de ellas no debería exceder los 60 minutos reloj. 
Para dotar de cierta heterogeneidad a la conformación de cada grupo focal, se podrían emplear algunos (o la totalidad) de los siguientes criterios de segmentación:

1. Para el caso de los docentes, procurar la participación de individuos con distinto/a: a) departamento de desempeño (por ejemplo Administración, Economía, Contabilidad), b) función (por ejemplo, docencia, investigación, transferencia tecnológica), c) género, d) grupo etario, e) jerarquía del cargo, f) dedicación horaria (por ejemplo, tiempo completo, tiempo parcial), g) afiliación política.

2. Para el caso de los estudiantes, procurar la participación de individuos: a) de diferentes carreras, b) con desigual grado de avance, c) con distinta situación ocupacional.

3. Para el caso del personal de apoyo, considerar la participación de individuos con distinto: a) agrupamiento o función (por ejemplo, personal administrativo, personal de maestranza, personal de soporte técnico), b) grupo generacional, c) afiliación política.

Se desaconseja la incorporación de integrantes de la Dirección dentro del grupo focal, pues podrían condicionar las respuestas del resto de los participantes.

Implicancias para la cultura organizacional. El empleo de dinámicas focales puede resultar sumamente útil para: a) el entendimiento y la interpretación de artefactos; b) la identificación de informantes clave; c) el descubrimiento de nuevos artefactos (por ejemplo, mitos, rituales, normas, prácticas) y símbolos menos accesibles para el observador a través de las estrategias anteriormente discutidas; d) la identificación preliminar de subgrupos y subculturas, así como los criterios que los demarcan; e) la exploración de valores organizacionales y grupales, entre otros.

Implicancias para la identidad organizacional. Puesto que esta herramienta habilita un mayor (aunque aún moderado) intercambio entre el investigador y los participantes, es posible comenzar a explorar qué es lo que éstos opinan acerca del conjunto de atributos más distintivos, durables y permanentes de la universidad a la que pertenecen.

Al discutir la revisión del estatuto y las comunicaciones formales de la Dirección a los integrantes se aludió a la influencia de aquellas en la construcción de sentido, proceso denominado sensegiving. Mediante la realización de focus groups el investigador es capaz de indagar, principalmente, en los procesos de construcción de significado compartido definidos previamente como sensemaking.

En la elaboración del guión, el investigador podría hallar útil incorporar algunos o la totalidad de los siguientes interrogantes: a) si tuvieran que imaginar la universidad de aquí a diez años, ¿la visualizan similar a la actual?, b) ¿cuáles serían sus principales cambios?, c) ¿podrían pensar en algunos principios que representan a esta universidad? (procurar que su emergencia sea espontánea), d) ¿̇en qué acciones concretas podemos observar tales principios? (emergencia espontánea), e) ¿creen que estos principios han cambiado a través del tiempo?, f) ¿qué principios creen que esta universidad debería sostener? (en caso de no emerger de manera espontánea sugerir algunos como: calidad académica, inclusión, democracia educativa, etcétera), g) ¿les parece que los procedimientos de gestión son y han sido coherentes con los valores mencionados?, h) cpueden enunciar algunos de esos procedimientos en los que verifican mayor congruencia/incongruencia?, i) ¿recuerdan alguna decisión o propuesta que haya colisionado con los valores mencionados? En caso de que el estudio se encuentre centrado en una sola escuela/facultad dentro de la universidad, puede ser útil solicitar a los participantes que piensen en torno a: facultades que creen que son similares a la estudiada, facultades que creen que son muy diferentes, principios que creen que se mantienen invariantes y son compartidos por la universidad en su conjunto. 
Implicancias para la imagen organizacional. Para Mintzberg (1992), toda organización posee un público portador de intereses, el cual puede ser clasificado en: a) grupos tan generales como las familias o los líderes de opinión pública; b) grupos con intereses especiales, como las sociedades científicas cuyo principal fin estatuido es promover el desarrollo del conocimiento en la disciplina de la que se trate; c) el gobierno en todas sus facetas. Mediante el empleo de esta herramienta es posible explorar: quiénes son los agentes considerados por los participantes como más relevantes (por ejemplo, el mercado laboral, la Academia, los Colegios Profesionales), así como la percepción que los participantes poseen de la imagen que los agentes externos tienen de la universidad.

\section{Las entrevistas grupales}

A diferencia de lo planteado para los focus groups, en donde se aconsejó que la composición de cada dinámica estuviera dotada de un cierto grado de heterogeneidad que permita observar la interacción acontecida entre miembros de diferentes subgrupos sociales, en las entrevistas grupales se pretenderá que cada grupo sea relativamente homogéneo. No obstante lo indicado respecto de la composición del grupo, esta herramienta comparte con el focus group la posibilidad de reunir evidencia espontánea producto de la interacción. En este sentido, puede ser útil conducir entrevistas grupales con investigadores, con docentes part-time, con aquellos que realizan transferencia tecnológica, con representantes de un cierto agrupamiento del personal de apoyo, entre muchos otros. La información relevada en el item P15 del cuestionario cuantitativo, así como la obtenida a través del empleo de otras herramientas, brinda orientación en la elección de los criterios a tener en cuenta en la conformación de los grupos de cada entrevista.

Implicancias para la cultura organizacional. Las entrevistas grupales son recomendadas como la herramienta por excelencia para la identificación y estudio de subculturas. Permiten al investigador observar a representantes de una misma unidad de trabajo interactuar entre sí e identificar elementos culturales distintivos de un grupo, por ejemplo: prácticas y formas usuales de hacer las cosas dentro de la unidad, valores grupales, normas deseadas de comportamiento, sanciones sociales aplicadas como resultado de percepciones de violación a las pautas del grupo, etcétera.

Implicancias para la identidad organizacional. $\mathrm{Al}$ tratarse de un grupo pequeño de participantes (usualmente entre tres y cinco) puede aprovecharse la instancia para reunir evidencia adicional que oriente al observador en la interpretación de elementos que hubieran surgido en etapas anteriores.

Implicancias para la imagen organizacional. Se reiteran las observaciones indicadas en el punto anterior.

\section{Las entrevistas en profundidad}

La entrevista en profundidad consiste en una interacción verbal cara a cara constituida por preguntas y respuestas orientadas a una temática u objetivos específicos (Oxman, 1998). Por ello, y de manera previa al relevamiento, se sugiere la confección de una guía de pautas o guión flexible que reúna los tópicos en torno a los cuales se efectuará la indagación. A su vez, la naturaleza flexible de la entrevista en profundidad le permite al observador la incorporación de elementos no planeados que motiven la emergencia espontánea de nuevas categorías de análisis conforme se desarrolla la conversación.

A través de la pregunta, el observador buscará relevar opiniones (expresan ideas, sentimientos, prejuicios del sujeto hacia un proceso, objeto o sujeto/s), actitudes (muestran predisposiciones o tendencias a la acción del sujeto hacia un proceso, objeto o sujeto/s), acciones (describen la participación del sujeto en procesos o interacciones), representaciones (evidencian estructuras mentales asociadas a un proceso, objeto o sujeto/s) y manifestaciones (refieren a expresiones culturales, componentes de imaginarios sociales, jergas grupales y/o institucionales; Fraire y Scribano, 2008). 
El empleo de las técnicas discutidas hasta el momento habrá orientado al observador lo suficiente en la selección de los informantes clave que deben ser entrevistados. Incluso, la participación de algunos de ellos en instancias previas no invalida su participación en esta fase. Puesto que en la entrevista en profundidad sólo participan el investigador y el sujeto entrevistado, ésta le ofrece al entrevistado un clima de mayor privacidad que las técnicas de participación colectiva, situación que favorece la discusión de tópicos relativamente sensibles a la presión de grupo. Por ello, puede constituir la herramienta más apropiada para reunir evidencia sobre los miembros de la Dirección de la universidad (por ejemplo, rector, decanos, secretarios).

Con relación al tópico particular de este artículo, la entrevista en profundidad puede resultarle de gran utilidad al observador por varios motivos. En primer lugar, el análisis del discurso de los entrevistados le permite interpretar y dar sentido a la evidencia reunida en instancias anteriores. En segundo lugar, la naturaleza altamente flexible de la entrevista en profundidad también le facilita la identificación de categorías no previstas hasta el momento o la indagación en aquellas con mayor nivel de especificidad. En tercer lugar, se configura como la herramienta por excelencia para la búsqueda y comprensión de los elementos más tácitos y subyacentes de la cultura organizacional (su esencia). Finalmente, le permite al investigador hipotetizar e indagar en las relaciones que subyacen entre la cultura, la identidad y la imagen organizacional.

\section{Consideraciones finales}

El presente artículo tuvo por objeto la construcción de una metodología para el estudio de la cultura, la identidad y la imagen organizacional en una institución universitaria, sustentada en una revisión profunda de la literatura y en la experiencia sistematizada de los autores a través de sus años de estudio del sistema universitario argentino. Se coincide con la postura dominante en la literatura de que la complejidad inherente a los fenómenos aquí discutidos les exige a los investigadores el empleo combinado de diversidad de estrategias, entre las que se destacan la observación participante, la revisión de fuentes documentales, los talleres, los focus groups, las entrevistas grupales y las entrevistas en profundidad.

Puesto que la metodología aquí propuesta ha sido construida con el propósito de brindar orientación a investigadores o a practitioners interesados en la temática, se reconoce que adaptaciones adicionales deberán efectuarse a la misma para ajustarla a los objetivos específicos del estudio. Como se mencionó oportunamente en este trabajo, la propuesta de triangular la información relevada a través de múltiples técnicas se sustenta en la hipótesis de mayor desconocimiento por parte del observador de la realidad organizacional bajo estudio y en la necesidad de que se adentre lo más posible en la misma a los efectos de rastrear los elementos más tácitos y subyacentes que definen la cultura, la identidad y la imagen organizacional, así como la complejidad de las relaciones que se suscitan entre estos tres fenómenos. Se admite que alguna o varias de las etapas aquí sugeridas podrían ser omitidas cuando existiera un mayor nivel de contacto o inmersión del observador en la organización bajo análisis. Sin embargo, se asume el riesgo de no ser capaz de ver lo que en realidad ocurre y que los propios prejuicios sesguen las interpretaciones.

Como parte de la metodología propuesta en este trabajo, también se presentó un Cuestionario de Cultura, Identidad e Imagen Organizacional, el cual se encuentra a disposición del lector en la sección Anexo. El mismo resulta genérico y requerirá de adaptaciones con la finalidad de ajustarlo a las necesidades particulares del revelamiento o a la idiosincrasia particular de la organización universitaria estudiada.

Se reitera la necesidad de que quienes participan en la gestión de universidades posean una comprensión profunda de la cultura organizacional, 
fundamentalmente por construir un fuerte condicionante en la toma de decisiones en todos los niveles. Como se ha enfatizado, al proveer a los miembros de un marco de referencia socialmente compartido e inconsciente que los orienta en su forma de percibir, interpretar y reaccionar a los eventos que ocurren en la organización, también influye fuertemente en la conformación de la identidad y la imagen organizacional y, en definitiva, en la racionalidad individual, grupal y organizacional que subyace a la hora de pensar alternativas y sus restricciones (lo posible), proceder a su evaluación (lo mejor), definir qué aspectos deben ser priorizados (lo deseable) y cuáles deben ser evitados (lo prohibido), considerar cuáles actores deben ser partícipes del proceso, etcétera. Como resultado, una noción sistémica de la cultura organizacional y sus relaciones resulta fundamental si se pretende la adopción de decisiones que se encuentren sujetas al menor nivel posible de resistencia, conflicto y antagonismo.

Una potencial línea de investigación en la temática consiste en el estudio de la identidad de una organización educativa cuando sus participantes perciben la emergencia de eventos amenazadores a la identidad (por ejemplo, una modificación del estatuto, un cambio en la normativa estatal que regula a las entidades educativas), similar al estudio conducido por Ravasi y Schultz (2006) en el ámbito privado. También podría investigarse el rol de la cultura organizacional como un condicionante en la toma de decisiones estratégicas, tácticas y operativas en la universidad. Finalmente, el estudio de los mecanismos y sistemas de gobierno en organizaciones de educación superior y su grado comparativo de efectividad también constituye una atractiva línea de investigación. 


\section{Anexo}

\section{Cuestionario de cultura, identidad e imagen organizacional ${ }^{5}$}

Estimado miembro de la comunidad universitaria, nos encontramos efectuando un relevamiento acerca de los aspectos más característicos de nuestra universidad y para ello sería enormemente enriquecedor contar con su opinión. Le recordamos que sus respuestas son anónimas, por lo que no debe consignar en este formulario dato identificatorio alguno. Le agradecemos enormemente el tiempo invertido en responder este cuestionario.

\section{Datos clasificatorios}

C1. Sexo

- Masculino

- Femenino

C2. Fecha de nacimiento (especifique)

C3. Rol

- Estudiante de Grado

- Estudiante de Postgrado

- Docente

- Personal Universitario

\section{Sólo si es docente:}

C4.1.1. Cargo (listar jerarquías según corresponda)

C4.1.2. Actividades que desempeña en la universidad, además de la docencia:

- Investigación

- Transferencia

- Gestión

C4.1.3. Condición:

- Cargo vitalicio

- Temporal

- Ad honorem

C4.1.4. Dedicación

- Part-time

- Full-time

C4.1.5. Antigüedad docente en esta universidad (especifique):

C4.1.6. Área/Departamento de desempeño (listar Departamento según corresponda):

\section{Sólo si es Estudiante de Grado o Postgrado}

C4.2.1. Carrera (listar carreras según corresponda):

C4.2.2. Año de ingreso a la universidad (especifique):

\section{Sólo si es parte del Personal de Apoyo}

C4.3.1. Categoría (especifique):

C4.3.2. Antigüedad (especifique):

C4.3.3. Agrupamiento

- Soporte técnico

- Maestranza

- Administrativo

- Otros (especifique):

Prequntas de relevamiento

P1. ¿Qué acontecimientos considera que han marcado la historia de nuestra universidad?

P2. ¿Usted presenció dicho acontecimiento?

- $\mathrm{Si}$

- No

- No contesta

\footnotetext{
${ }^{5}$ El presente cuestionario puede ser empleado, reproducido y/o adaptado en su totalidad o en cualquiera de sus partes sin necesidad de contar con previo consentimiento por parte de sus autores, siempre y cuando se haga reconocimiento expreso de autoría.
} 
P3. Sólo si NO a P2, ¿cómo tomó conocimiento de aquel? (puede marcar más de una opción)

- Por compañeros de trabajo

- Por fuentes documentales

- A través de comunicaciones formales (institucionales)

- Otros(especifique):

P4. Enuncie hasta cinco (5) valores que usted cree priman en la toma de decisiones de nuestra universidad.

P5. A continuación se le presenta una tabla compuesta por dos (2) partes. En la parte 1, se le solicita que califique una lista de aspectos en función de cuan presente Usted cree que se encuentra cada uno en nuestra universidad, asignando un número del 1 (nada presente) al 5 (totalmente presente). En la parte $\mathbf{2}$, se le pide que compare cada aspecto de la lista con otras instituciones en función de su grado de similitud, asignando un puntaje del 1 (en este aspecto la universidad y la institución comparada son totalmente distintos) al 5 (en este aspecto la universidad y la institución comparada son totalmente iguales). En caso de no desear responder, puede dejar la celda de la tabla vacía.

\begin{tabular}{|c|c|c|c|}
\hline \multirow[b]{2}{*}{ Aspecto } & \multirow{2}{*}{$\begin{array}{c}\text { Parte } 1 \\
\begin{array}{c}\text { Presencia en nuestra } \\
\text { universidad }\end{array}\end{array}$} & \multicolumn{2}{|c|}{ Parte 2} \\
\hline & & $\begin{array}{l}\text { Institutos de formación } \\
\text { no universitaria }\end{array}$ & $\begin{array}{l}\text { Otras universidades } \\
\text { locales }\end{array}$ \\
\hline \multicolumn{4}{|l|}{ Igualdad } \\
\hline \multicolumn{4}{|l|}{ Respeto } \\
\hline \multicolumn{4}{|l|}{ Cooperatividad } \\
\hline \multicolumn{4}{|l|}{ Armonía en los vínculos } \\
\hline \multicolumn{4}{|l|}{ Vinculación con la comunidad } \\
\hline \multicolumn{4}{|l|}{ Tolerancia a la diversidad } \\
\hline \multicolumn{4}{|l|}{ Orientación a la calidad } \\
\hline \multicolumn{4}{|l|}{ Orientación a la equidad en las decisiones } \\
\hline \multicolumn{4}{|l|}{$\begin{array}{l}\text { Orientación a la pertinencia (contenidos de las } \\
\text { asignaturas, la oferta, las temáticas de proyectos, } \\
\text { etcétera) }\end{array}$} \\
\hline \multicolumn{4}{|l|}{ Orientación a la innovación } \\
\hline \multicolumn{4}{|l|}{ Orientación a la eficiencia } \\
\hline \multicolumn{4}{|l|}{ Participación } \\
\hline \multicolumn{4}{|l|}{ Formación integral del graduado } \\
\hline \multicolumn{4}{|l|}{$\begin{array}{l}\text { Excelencia de la formación del cuerpo de docentes } \\
\text { e investigadores }\end{array}$} \\
\hline Exigencia en el sistema de evaluación & & & \\
\hline
\end{tabular}

P6. Indique hasta tres (3) valores o principios que usted percibe que se encuentran presentes (hoy) en nuestra universidad y que Usted no comparte.

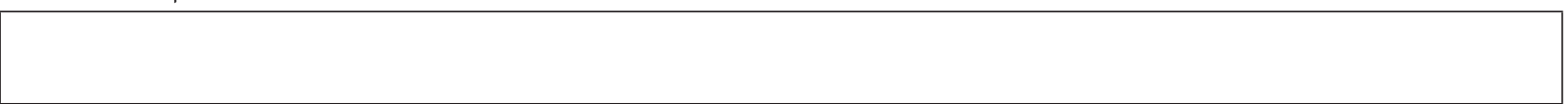

P7. ¿Cree que los valores que actualmente sostiene nuestra universidad contribuyen al cumplimiento de su misión?

- $\quad \mathrm{Si}$

- No

- No sabe/no contesta

P8. Sólo si NO en P7, ¿podría indicar algún ejemplo concreto? 
P9. Teniendo en cuenta los valores y principios que usted considera que se encuentran presentes en nuestra universidad, se le pide que recuerde a alguna persona que hoy o en el pasado haya sido representativa de los valores mencionados.

\begin{tabular}{|l|l|l|}
\hline Persona & Rol que desempeña/desempeñó & $\begin{array}{c}\text { Valor que usted cree que encarna/ha } \\
\text { encarnado }\end{array}$ \\
\hline & & \\
\hline & & \\
\hline
\end{tabular}

P10. Mencione los tres (3) aspectos más característicos de nuestra universidad que usted cree que han permanecido más invariantes (sin cambios) a lo largo del tiempo.

P11. Mencione tres (3) rasgos que, a su parecer, más han cambiado a lo largo del tiempo.

P12. De la siguiente lista de rasgos se le solicita que califique cada uno en función de su grado de permanencia a través del tiempo en nuestra universidad, asignándole un puntaje del 1 (el atributo ha variado enormemente a través del tiempo) al 5 (el atributo ha sido invariante a través del tiempo).

\begin{tabular}{|c|c|}
\hline Aspecto & Grado de permanencia \\
\hline Igualdad & \\
\hline Respeto & \\
\hline Cooperatividad & \\
\hline Armonía en los vínculos & \\
\hline Vinculación con la comunidad & \\
\hline Tolerancia a la diversidad & \\
\hline Orientación a la calidad & \\
\hline Orientación a la equidad en las decisiones & \\
\hline Orientación a la pertinencia (contenidos, las carreras, temáticas de proyectos, etcétera) & \\
\hline Orientación a la innovación & \\
\hline Orientación a la eficiencia & \\
\hline Participación & \\
\hline Formación integral del graduado & \\
\hline Excelencia de la formación del cuerpo de docentes e investigadores & \\
\hline Exigencia en el sistema de evaluación & \\
\hline
\end{tabular}

P13. Marque con una X los agentes que Usted considera que son clave para el desarrollo de las actividades de nuestra universidad (puede marcar más de una opción).

- Comunidad empresaria

- Organizaciones no gubernamentales

- Colegios/Asociaciones Profesionales

- Comunidad en general

- Comunidad científica (revistas científicas de elevado prestigio y sus cuerpos editoriales, referentes disciplinares de otras Instituciones de Ciencia y Técnica/Educativas)

- Estado

- Otros (especifique): 
P14. Califique del 1 (nivel más bajo) al 5 (nivel más alto) la imagen que usted cree que los siguientes agentes tienen de nuestra universidad.

\begin{tabular}{|l|l|l|l|l|l|}
\hline \multicolumn{1}{|c|}{ Aspecto } & Mercado & Comunidad & Academia & Estado & $\begin{array}{c}\text { Otras } \\
\text { instituciones } \\
\text { educativas }\end{array}$ \\
\hline Imagen general & & & & & \\
\hline $\begin{array}{l}\text { Calidad de los docentes } \\
\text { e investigadores }\end{array}$ & & & & & \\
\hline $\begin{array}{l}\text { Competencias de los } \\
\text { estudiantes }\end{array}$ & & & & & \\
\hline $\begin{array}{l}\text { Calidad de los } \\
\text { graduados }\end{array}$ & & & & & \\
\hline $\begin{array}{l}\text { Productos de } \\
\text { investigación }\end{array}$ & & & & & \\
\hline $\begin{array}{l}\text { Vínculos con la } \\
\text { comunidad }\end{array}$ & & & & & \\
\hline
\end{tabular}

P15. Dentro de las organizaciones, las personas tienden a asociarse con otras conformando grupos sociales. Se le pide que piense algunos ejemplos de subgrupos sociales que usted percibe que existen en Docentes, Estudiantes y Personal de Apoyo. Indique, para cada grupo, tres (3) causas por las cuales cree que éstos se conforman.

Considérese el siquiente ejemplo: en un hospital, aun dentro del cuerpo de médicos, podrían formarse subgrupos en función de su especialidad o de los turnos en los que trabajan.

Docentes:

\section{Estudiantes:}

Personal de apoyo:

Muchas gracias por su enorme colaboración. 


\section{Referencias}

Albert, Stuart, David Whetten (1985), "Organizational identity", en Research in Organizational Behavior, núm. 7, pp. 263-295.

Corley, Kevin, Celia Harquail, Michael Pratt, Mary Ann Glynn, Marlene Fiol y Mary Jo Hatch (2006), "Guiding organizational identity through aged adolescence", en Fournal of Management Inquiry, vol. 15, núm. 2, pp. 85-99.

Deal, Terrence y Allan Kennedy (1982), Corporate cultures: the rites and rituals of organizational life, Mass, AddisonWesley.

Dutton, Jane y Janet Dukerich (1991), "Keeping an eye on the mirror: Image and identity in organizational adaptation", en Academy of Management fournal, vol. 34, núm. 3, pp. 517-554.

Fraire, Vanina y Adrián Scribano (2008), "El grupo de discusión: posibilidades y estrategias", en El proceso de investigación social cualitativo, Buenos Aires, Prometeo libros.

Fumasoli, Tatiana y Bjorn Stensaker (2013), "Organizational studies in higher education: A reflection on historical themes and prospective trends", en Higher Education Policy, vol. 26, núm. 4, pp. 479-496.

Gioia, Dennis, Aimee Hamilton y Shubha Patvardhan (2014), "Image is everything: reflections on the dominance of image in modern organizational life", en Research in Organizational Behavior, vol. 34, núm. 1, pp. 129-154.

Gioia, Dennis, Shubha Patvardhan, Aimee Hamilton y Kevin Corley (2013), "Organizational identity formation and change", en Academy of Management Annals, vol. 7, núm. 1, pp. 123-193.

Gioia, Dennis, Majken Schultz y Kevin Corley (2000), "Organizational identity, image, and adaptive instability", en Academy of Management Review, vol. 25, núm. 1, pp. 63-81.

Giorgi, Simona, Christi Lockwood y Mary Ann Glynn (2015), "The many faces of culture: making sense of 30 years of research on culture in organization studies", en The academy of Management Annals, vol. 9, núm. 1, pp. 1-54.
Harris, Stanley (1994), "Organizational culture and individual sensemaking: a schema-based perspective", en Organization Science, vol. 5, núm. 3, pp. 309-321.

Hatch, Mary Jo (1997), "The dynamics of organizational culture", en Academy of Management Review, vol. 18, núm. 4, pp. 657-693.

Hatch, Mary Jo y Majken Schultz (2002), "The dynamics of organizational identity", en Human Relations, vol. 55, núm. 8, pp. 989-1018.

Hatch, Mary Jo y Majken Schultz (1997), "Relations between organizational culture, identity and image", en European fournal of Marketing, vol. 31, núm. 5/6, pp. 356-365.

Hofstede, Geert, Michael H. Bond y Chung-leung Luk (1993), "Individual perceptions of organizational cultures: A methodological treatise on levels of analysis", en Organization Studies, vol. 14, núm. 4, pp. 483-503.

Humphreys, Michael y Andrew Brown (2002), "Narratives of organizational identity and identification: a case study of hegemony and resistance", en Organization Studies, vol. 23, núm. 3, pp. 421-447.

Martin, Joanne, Peter Frost y Oliver O’Neill (2006), "Organizational culture: beyond struggles for intellectual dominance", en The Handbook of Organization Studies, Londres, SAGE Publications Ltd, pp. 725-753.

Mintzberg, Henry (1992), Structure in fives: Designing effective organizations, New Jersey, Prentice Hall.

Oxman, Claudia (1998), La entrevista de investigación en Ciencias Sociales, Buenos Aires, EUDEBA.

Ravasi, Davide y Majken Schultz (2006), "Responding to organizational identity threats: exploring the role of organizational culture", en Academy of Management Fournal, vol. 49, núm. 3, pp. 433-458.

Ravasi, Davide (2016), "Organizational identity, culture, and image", en The Oxford Handbook of Organizational Identity, Oxford, Oxford University Press.

Ribes Pons, Ángel (1997), "La cultura de la empresa: cómo actuar en ella y desde ella", en Deusto Business Review, núm. 79, pp. 10-49. 
Sackmann, Sonja (1992), "Culture and subcultures: an analysis of organizational knowledge", en Administrative Science Quarterly, vol. 37, núm. 1, pp. 140-161.

Schein, Edgar (2010), Organizational culture and leadership, San Francisco, John Wiley \& Sons.

Schein, Edgar (1984), "Coming to a new awareness of organizational culture", en Sloan Management Review, vol. 25, núm. 2, pp. 3.

Schneider, Benjamin, Mark Ehrhart y William Macey (2013), "Organizational climate and culture", en Annual Review of Psychology, vol. 64, núm. 1, pp. 361-388.

Stensaker, Bjorn (2015), "Organizational identity as a concept for understanding university dynamics", en Higher Education, vol. 69, núm. 1, pp. 103-115.
Stensaker, Bjorn, Jussi Välimaa y Claudia Sarrico (2012), Managing reform in universities: the dynamics of culture, identity and organisational change, Londres, Palgrave Macmillan.

Tierney, William (2008), The impact of culture on organizational decision-making: theory and practice in higher education, Stylus Publishing.

Tierney, William (1988), "Organizational culture in higher education: defining the essentials", en The Journal of Higher Education, vol. 59, núm. 1, pp. 2-21.

Whetten, David (2006), "Albert and Whetten revisited: strengthening the concept of organizational identity", en Fournal of Management Inquiry, vol. 15, núm. 3, pp. 219-234.

\section{Cómo citar este artículo:}

Pujol-Cols, Lucas J. y Mariana Foutel (2018), “Cultura, identidad e imagen organizacional: desarrollo de una metodología para su estudio en la Universidad", en Revista Iberoamericana de Educación Superior (RIES), México, UNAM-IISUE/Universia, vol. IX, Núm. 26, pp. 64-86, [consulta: fecha de última consulta]. 